\title{
Dirac-bracket quantization of chiral scalar two-dimensional QED
}

\author{
E. Abdalla \\ Instituto de Física, Universidade de São Paulo, C.P. 20516, 01498 São Paulo, São Paulo, Brazil \\ M. C. B. Abdalla, F. P. Devecchi, and A. Zadra \\ Instituto de Física Teórica, Universidade Estadual Paulista, Rua Pamplona, 145, 01405 Săo Paulo, São Paulo, Brazil \\ (Received 23 March 1988; revised manuscript received 11 July 1988)
}

\begin{abstract}
We discuss the interaction of two-dimensional chiral bosons with an electromagnetic field, quantizing the system via the Dirac procedure. A quantum theory is obtained; however, due to the mass generation the system is consistent only if further constraints are imposed. In such a case, the electromagnetic field is trivial. The role of the gauge Jackiw-Rajaraman mass parameter is also discussed.
\end{abstract}

\section{INTRODUCTION}

Frequently, symmetries do not survive the quantization procedure. A major example of such an anomalous conservation law is given by chiral gauge theories, ${ }^{1}$ where gauge invariance is broken at the quantum level. In the case of two-dimensional spinor QED and QCD, there have been several proposals ${ }^{2,3}$ for canceling anomalies introducing Wess-Zumino (WZ) terms. ${ }^{4}$ Consistent quantization of the chiral Schwinger model has been achieved by Jackiw and Rajaraman, ${ }^{5}$ who showed the existence of a one-parameter family of chiral Schwinger models. Girotti, Rothe, and Rothe ${ }^{6}$ quantized the bosonized theory with the Wess-Zumino term via the Dirac procedure $^{7}$ for constrained systems. Thus two-dimensional QED $\left(\mathrm{QED}_{2}\right)$ has been shown to depend on the value of the Jackiw-Rajaraman (JR) parameter, and may be preserved as two types of theories, with either two or four constraints.

The non-Abelian case was considered in Refs. 8 and 9. In the former reference, the author based his discussion on Witten's ${ }^{10}$ bosonization prescription using Coleman's arguments. 11 In the latter work, a "first-principles" derivation of the effective action involving the $\mathrm{WZ}$ term was given, and thereafter the Dirac quantization procedure was applied. As in the Abelian case there were two cases, involving either two or four constraints, the latter in a distinguished value of the JR $a$ parameter.

In this paper we discuss the coupling of chiral bosons to an Abelian gauge field.

Chiral bosons have been recently studied by a number of authors. ${ }^{12,13}$ The theory has been consistently quantized $^{14}$ using a nonlocal Lagrangian and a local commutation rule, or equivalently a local Lagrangian but a nonlocal commutation rule.

It was also shown that the system may be quantized via the Dirac procedure. ${ }^{15}$

In Sec. II we consider the coupling to a gauge field, writing the (classically) gauge-invariant action, and the constraints. In Sec. III we derive the constraint algebra and quantize the theory via Dirac brackets. Equations of motion are also discussed, as well as the particular value $a=0$. In Sec. IV we draw conclusions.

\section{CHIRAL SCALAR QED 2}

A pure left-moving bosonic field $\phi\left(x_{0}-x_{1}\right)$ may be described by the Lagrangian

$$
\mathcal{L}=\frac{1}{2} \partial^{\mu} \phi \partial_{\mu} \phi
$$

with the constraint

$$
\partial_{-} \phi=0,
$$

which may be canonically quantized, as far as we are able to deal with the second-class constraint

$$
\Omega(x)=\pi(x)-\phi^{\prime}(x) \approx 0 .
$$

Some authors proposed to substitute the above constraints by the first-class constraint ${ }^{12}$

$$
L(x)=\Omega(x)^{2},
$$

which is dealt by the action ${ }^{16}$

$$
\mathcal{L}=\frac{1}{2} \sqrt{-g} g^{\alpha \beta} \partial_{\alpha} \phi \partial_{\beta} \phi
$$

with the gravity field truncated as

$$
\sqrt{-g} g^{\alpha \beta}=\left[\begin{array}{cc}
0 & 1 \\
1 & -\lambda^{--}
\end{array}\right],
$$

which leaves us with the expression

$$
\mathcal{L}=\partial_{+} \phi \partial_{-} \phi-\frac{1}{2} \lambda^{--}\left(\partial_{-} \phi\right)^{2},
$$

where the doubly self-dual field $\lambda^{--}$realizes the firstclass constraint. A description of the model by means of the second-class constraint (2.2) is feasible using the Dirac method. The constraint satisfies

$$
\{\Omega(x), \Omega(y)\}=-2 \delta^{\prime}(x-y),
$$


which determines a second-class system (although the number of constraints seems to be odd, ${ }^{7}$ going to a discretized version of space one sees that an antisymmetric matrix emerges ${ }^{17}$ ).

Thus one derives the commutator ${ }^{14}$

$$
[\phi(x), \phi(y)]=-\frac{i}{4} \epsilon(x-y) \text {. }
$$

The interaction of the above model with a gauge field was proposed in Ref. 12 . We write the Lagrange density

$\mathcal{L}=\partial_{+} \phi \partial_{-} \phi+A_{-} \partial_{+} \phi-A_{+} \partial_{-} \phi-\frac{1}{4} F_{\mu \nu}^{2}+\frac{1}{2} a A^{\mu} A_{\mu}$,

where the last term has an arbitrary parameter $a$ related to the gauge symmetry breaking, and should be put in, to take into account the renormalization arbitrariness.

\section{DIRAC QUANTIZATION OF THE MODEL}

Canonical quantization of Lagrangian (2.9) may be achieved defining the momenta

$$
\begin{aligned}
& \pi=\dot{\phi}-A_{1}, \\
& \pi_{0}=0, \\
& \pi_{1}=A_{0}^{\prime}-\dot{A}_{1}=F_{10},
\end{aligned}
$$

with the canonical Hamiltonian given by

$$
\begin{gathered}
H_{c}=\int d x^{1}\left[\frac{1}{2} \pi_{1}^{2}+\frac{1}{2}\left(\pi+A_{1}\right)^{2}+\frac{1}{2} \phi^{2}+A_{0}\left(\pi_{1}^{\prime}-\phi^{\prime}\right)\right. \\
\left.-\frac{1}{2} a\left(A_{0}^{2}-A_{1}^{2}\right)\right],
\end{gathered}
$$

with the primary constraint

$$
\Omega_{1}=\pi_{0}(x) \approx 0 \text {. }
$$

The chirality constraint in the canonical formalism is given by

$$
\widetilde{\Omega}(x)=\pi(x)-\phi^{\prime}(x)+A_{1}(x) \approx 0 .
$$

However, we will refrain from imposing (3.4) at the beginning, since quantizing the system with (3.3) and (3.4) leads to a further constraint $\pi_{1} \approx 0$. This condition is obtained a posteriori, considering the bosonic equation of motion in terms of Dirac brackets, after a long, albeit straightforward calculation using the Dirac procedure.

In order to clarify the issue, we first consider the system using (3.3).

Time conservation of $\Omega_{1}$ leads to the Gauss law

$$
\Omega_{2}(x)=-\pi_{1}^{\prime}+\phi^{\prime}+a A_{0} \approx 0 .
$$

The system constrained by $\Omega_{1}$ and $\Omega_{2}$ is second class, with a total Hamiltonian given by

$$
\begin{aligned}
H_{T}=\int d x^{1} & \left(\frac{1}{2}\left(\pi+A_{1}\right)^{2}+\frac{1}{2} \pi_{1}^{2}+\frac{1}{2} \phi^{\prime 2}+\frac{1}{2 a}\left(-\pi_{1}^{\prime}+\phi^{\prime}\right)^{2}\right. \\
& \left.+\frac{a}{2} A_{1}^{2}\right)
\end{aligned}
$$

with the nonvanishing Dirac brackets

$$
\begin{aligned}
& \{\phi(x), \pi(y)\}_{\mathrm{DB}}=\delta(x-y), \\
& \left\{A^{1}(x), \pi_{1}(y)\right\}_{\mathrm{DB}}=\delta(x-y),
\end{aligned}
$$

and the identities

$$
\begin{aligned}
& \pi_{0}=0, \\
& A_{0}=\frac{1}{a}\left(\pi_{1}^{\prime}-\phi^{\prime}\right) .
\end{aligned}
$$

Let us now add the chiral constraint

$$
\widetilde{\Omega}_{1}(x)=\pi+A_{1}-\phi^{\prime} \approx 0 .
$$

If this is the only constraint to be imposed, we get, using the Dirac procedure, the equation of motion

$$
\dot{\phi}=\phi^{\prime}+\frac{1}{4} \int d y \pi^{1}(y) \epsilon(x-y),
$$

which from the point of view of taking $\phi$ as being a right-mover field is erroneous, unless we impose the further constraint $\pi_{1}=0$. For this reason we impose (3.9) as an external constraint to the equations of motion in order to achieve a consistent system. In order that $\widetilde{\Omega}_{1}$ be constant in time, with the Hamiltonian (3.6), we get the new constraint

$$
\widetilde{\Omega}_{2}=\pi+(1+a) A_{1} \approx 0 .
$$

On the other hand, the equations of motion for $\phi, \pi$, and $A_{1}$,

$$
\begin{aligned}
& \dot{\phi}=\pi+A_{1}, \\
& \dot{\pi}=\phi^{\prime \prime}+\frac{1}{a}\left(-\pi_{1}+\phi\right)^{\prime \prime}, \\
& \dot{A}_{1}=-\pi_{1}+\frac{1}{a}\left(\pi_{1}^{\prime \prime}-\phi^{\prime \prime}\right),
\end{aligned}
$$

imply

$$
\square \phi+\pi_{1}=0 \text {. }
$$

Since $\phi$ is a right-mover field, we obtain

$$
\widetilde{\Omega}_{3}=\pi_{1} \approx 0 \text {. }
$$

The system $\widetilde{\Omega}_{1}, \widetilde{\Omega}_{2}, \widetilde{\Omega}_{3}$ is consistent and does not generate any further constraint. An even better set of constraints is given by

$$
\begin{aligned}
& \hat{\Omega}_{1}(x)=\pi-\frac{1+a}{a} \phi^{\prime} \approx 0, \\
& \hat{\Omega}_{2}(x)=-\pi_{1} \approx 0, \\
& \hat{\Omega}_{3}(x)=\pi+(1+a) A_{1} \approx 0 .
\end{aligned}
$$

The Dirac matrix is readily computed, and we have the nonvanishing commutators:

$$
\begin{aligned}
& {[\phi(x), \phi(y)]=-i \hbar \frac{a}{4(1+a)} \epsilon(x-y),} \\
& {[\phi(x), \pi(y)]=\frac{i \hbar}{2} \delta(x-y),}
\end{aligned}
$$




$$
[\pi(x), \pi(y)]=i \hbar \frac{1+a}{2 a} \delta^{\prime}(x-y) .
$$

Further commutators are obtained using (3.15). We may write the Hamiltonian using now the constraints strongly

$$
H=\int d x^{1} \frac{1+a}{a} \phi^{2}
$$

From (3.16a) and (3.17) we get

$$
\dot{\phi}(x)=\phi^{\prime}(x)
$$

The system is well defined only for $a>0$ or $a<-1$.

The case $a=0$ must be treated separately, since further constraints arise. As it turns out, in that case the theory is trivial, since we eventually obtain $\phi=0$. This is the same result as the one obtained from (3.16a), making $a=0$.

\section{CONCLUSIONS}

The interaction of chiral bosons with a quantized electromagnetic field yields a theory where all gauge field degrees of freedom disappear due to constraints. The whole theory reduces to a single chiral boson, proportional to the Floreanini-Jackiw solution, the constant of proportionality being a function of the gauge-field mass parameter $a$. This is the only trace of the gauge-field interaction. A similar effect appears in the pure chiral boson with a Wess-Zumino term related to Siegel symmetry: ${ }^{18}$ as it turns out, the effect of the extra WZ term is trivial, due to constraints. $^{19}$

\section{ACKNOWLEDGMENTS}

The work of E.A. and M.C.B.A. was partially supported by Conselho Nacional de Desenvolvimento Científico e Tecnológico (Brazil). The work of F.P.D. and A.Z. was supported by Fundação de Amparo e Pesquisa do Estado de São Paulo (Brazil).
${ }^{1} \mathrm{R}$. Jackiw, in Relativity, Groups and Topology II, proceedings of the Les Houches Summer School, Les Houches, France, 1983, edited by R. Stora and B. DeWitt (Les Houches Summer School Proceedings, Vol. 40) (North-Holland, Amsterdam, 1984).

${ }^{2}$ L. D. Faddeev and S. L. Shatashvili, Phys. Lett. 167B, 225 (1986).

${ }^{3}$ O. Babelon, F. A. Schaposnik, and C. M. Viallet, Phys. Lett. B 177, 385 (1986); K. Harada and I. Tsutsui, ibid. 183, 311 (1986).

${ }^{4}$ J. Wess and B. Zumino, Phys. Lett. 37B, 95 (1971).

${ }^{5}$ R. Jackiw and R. Rajaraman, Phys. Rev. Lett. 54, 1219 (1985).

${ }^{6}$ H. O. Girotti, H. J. Rothe, and K. D. Rothe, Phys. Rev. D 33, 514 (1986).

${ }^{7}$ P. A. M. Dirac, Lectures in Quantum Mechanics (Yeshiva University, New York, 1964).
${ }^{8}$ R. Rajaraman, Phys. Lett. 154B, 305 (1985).

${ }^{9}$ E. Abdalla and K. D. Rothe, Phys. Rev. D 36, 3190 (1987).

${ }^{10}$ E. Witten, Commun. Math. Phys. 92, 455 (1984).

${ }^{11}$ S. Coleman, Phys. Rev. D 11, 2088 (1975).

12J. M. F. Labastida and M. Pernici, Nucl. Phys. B297, 557 (1988); Phys. Rev. Lett. 59, 2511 (1987).

${ }^{13}$ Y. Frishman and J. Sonnenschein, Nucl. Phys. B301, 346 (1988).

${ }^{14}$ R. Floreanini and R. Jackiw, Phys. Rev. Lett. 59, 1873 (1987).

${ }^{15}$ M. E. V. Costa and H. O. Girotti, Phys. Rev. Lett. 60, 1771 (1988).

${ }^{16}$ W. Siegel, Nucl. Phys. B238, 307 (1984).

${ }^{17}$ H. O. Girotti (private communication).

${ }^{18}$ C. Imbimbo and A. Schwimmer, Phys. Lett. B 193, 455 (1987).

${ }^{19}$ E. Abdalla, M. C. B. Abdalla, F. P. Devecchi, and A. Zadra, Phys. Lett. B (to be published). 